\title{
Clinical experiences of perioperative nursing Masters Students in selected Rwandan Referral Hospitals
}

\author{
David Ryamukuru ${ }^{1 *}$, Joselyne Mukantwari ${ }^{1}$, Lilian Omondi ${ }^{1}$, Gilbert Rutayisire Karonkano ${ }^{1}$, Leoncie \\ Uzikwambara $^{1}$, Emmanuel Munyaneza ${ }^{1}$, Eric Karera ${ }^{1}$, Thierry Uwera ${ }^{1}$, Felix Mbonera ${ }^{1}$, Christian Ntakirutimana ${ }^{1}$, \\ Philip Humure Nzamurambaho ${ }^{1}$, Jean Pierre Nsekambabaye ${ }^{1}$, Odile Umuhoza ${ }^{1}$, Eric Sindayigaya ${ }^{1}$, Madeleine \\ Mukeshimana $^{1}$, Donatilla Mukamana ${ }^{1}$
}

1. College of Medicine and Health Sciences, School of Nursing and Midwifery Sciences, University of Rwanda

*corresponding author: David Ryamukuru (daviramu@yahoo.fr)

\begin{abstract}
Background: Clinical experiences allow nursing students to apply theoretical knowledge into practice hence socialize with their future professional roles and responsibilities. This is a report of observations made by thirteen perioperative nursing students in the first cohort of Master of sciences in nursing training program during their practicum in selected Rwandan Referral Hospitals aimed at improving students' learning outcomes in future. Methods: Teams of four to five students were allocated to the operating theatres in three selected referral and teaching hospitals in Rwanda for thirteen weeks. At the end of their clinical placement, they wrote clinical reports that highlighted their observations informed by theoretical knowledge of perioperative nursing care using a structure, process and outcome format. These reports focused on the strengths and challenges observed. Results: Students were able to translate theoretical knowledge gained in class to clinical practice. Although the hospitals provided good learning opportunities in relation to the number of patients attended to and the various surgical treatments provided, the existing infrastructures, and care delivery systems, still need improvement. Conclusions: Hospital structure and processes helped the students to achieve their clinical learning objectives. Fostering strong collaboration of Universities, Hospitals and Ministry of Health will promote specialization in the emerging field of perioperative nursing in Rwanda.
\end{abstract}

Key words: clinical experiences, masters program students training, perioperative nursing, referral hospital.

\section{Introduction}

Perioperative nursing in Rwanda has been scaled up to the master's level as part of Ministry of Health and Human Resource for Health $(\mathrm{HRH})$ program. One of the aims of this program is to increase the number of the appropriately skilled and motivated health care providers in Rwanda. [1] In this regard, combined efforts between the University of Rwanda (UR), College of Medicine and Health Sciences, Ministry of Health, and consortium of USA Universities were made in 2015. The University of Rwanda, School of Nursing and Midwifery started Masters of sciences in Nursing in eight nursing specialities including perioperative nursing.[2] This nursing speciality deals with care of patient before, during and after surgery. The three selected referral hospitals were used to provide clinical experiences.

Effective teaching in nursing is vital to equipping future nurse professionals with required knowledge, attitudes and skills necessary for meeting patient needs[3] including perioperative nursing care. Clinical experience has been always an integral part of nursing education. It prepares students to translate theoretical knowledge into practice and to develop psychomotor skills, clinical reasoning and decision making.[3]

Clinical experience is acquired through practices in health care settings including the community, health centers, hospitals as well as specialized departments such as the operating theaters.[4] Clinical setting has been highlighted in literature as the space where nursing students are socialized into their future roles in the field of nursing they want to work after graduation. [4] The clinical learning experiences are directly influenced by the effectiveness of clinical teaching and clinical environment. This enables the nursing students to apply abstract concepts into specific and concrete situations that characterize the professional roles and values. [5]

Regarding the perioperative trainings, the delivery of perioperative patient care requires complex knowledge and skills to effect safe outcomes for the patients 
.This role requires nurses to be educated theoretically and clinically to gain required knowledge, attitude and skills in perioperative nursing.[6] The Literature reviewed show that the operating theatre can provide positive learning experiences to nursing students.[7] This is influenced by positive attitudes, knowledge and willingness of the staff to teach and socialize students with the operating theatre nurse practitioners' roles and responsibilities.[7]

The perioperative nurse is one who specializes in perioperative practice and who provides nursing care to the surgical patients throughout the continuum of surgical care[8].This continuum of surgical care involves preoperative, intra operative, and postoperative care. $[8,9]$ Perioperative nurses deliver comprehensive patient care during operative and other invasive procedures, using the framework of the nursing process. Nursing process has five phases which include assessment, diagnosis, planning, implementing care plan and evaluating outcomes of the care interventions. Perioperative nurses use all the five phases of nursing process perioperatively in order to promote recovery of health, prevent further injury or illness and facilitate coping with alterations in physical structure and function of operated patients. [9]

The perioperative nurse is in the unique and privileged position as she/he assists with surgical procedure and acts as the consciousness of the unconscious patient[6]. This requires a clinical experience to develop critical thinking, clinical judgment and clinical reasoning.[10]

In the period before 1970s, the area of perioperative nursing work was narrowed to operating room and the nurses' roles were confined to the intraoperative nursing care only hence these nurses were called operating room (OR) nurses.[8,9] The OR nurses were viewed to have no patients responsibility but have to take care of OR equipment.[9] In contrast, perioperative nursing of today involves the care that begins at the time the patient is informed about surgery, continue during operation and postoperative period until full recovery of the surgical patient at home.[9]

Perioperative nurses were not formally trained in Rwanda until 2015 when the University of Rwanda began the program at masters' level. [11] Prior to this time, the education of nurses in Rwanda that is traced back to 1940 s, was training the assistant midwives, then progressively to A3, A2 professional certificates. [11] The training at the University level was sustainably started after 1994 genocide against the Tutsi. The trained nurses were awarded advanced diploma (A1) in general nursing, midwifery and mental health. In 2002, bachelor degree in nursing program was started.[12] In the meantime, the nurses were receiving on-the-job training in perioperative nursing care once deployed to operating theatres.

Based on perioperative nursing history in Rwanda and the emerging global roles of perioperative nurses, there was no literature found that explored clinical experiences in perioperative nursing area in the Rwandan context. Therefore, this article highlights the clinical experiences of thirteen master's students of perioperative nursing, the first cohort in selected Rwandan referral hospitals.

\section{Methods}

Informed observation made by 13 perioperative masters students following thirteen weeks of clinical experiences (from May 3 to June 15, 2016 and from October 31 to December 7,2016$)$ were summarized to compile this report. The observations were guided by the theoretical knowledge in perioperative nursing. These students reported their clinical experiences in three referral hospitals in Kigali, which were University Teaching Hospital of Kigali (CHUK), King Faisal Hospital $(\mathrm{KFH})$ and Rwandan Military Hospital (RMH). Apart from KFH which is a private hospital, the other two are national public hospitals in Rwanda. Students were allocated in the operating theatres in groups of four to five. They had opportunities to visit surgical patients in the wards, to conduct preoperative assessment that informed individualized patients' theatre preparations, and later, participated in their surgeries as members of the surgical team. The nurses followed up the patients to the recovery room to ensure safe recovery from anesthesia and surgery before being discharged back to the wards.

Three students' reports from these referral hospitals were reviewed and their clinical experiences were analyzed through the lens of Avedis Donabedian's model of quality improvement. This model used triad evaluation based on structure, process and outcomes. [13] The analysis also focused on areas of strengths and areas of improvements.

\section{Key Clinical learning observations \\ 1. The structure of health care system}

The reports highlighted that among the three referral hospitals, two were still in the process of international accreditation and the students benefited from the process through better understanding of how the accreditation is done and what is required to be accredited. These hospitals had the basic needed infrastructures, funds and staff and served many patients with varied conditions. These hospitals had at least five operating rooms and postoperative care units (PACU) with at least four beds for each hospital. The hospitals also had the instruments, supplies and equipment available for various surgical disciplines including surgical specialties such as sophisticated delicate endoscopic and 
microsurgeries equipment and instruments that need special expertise for usage and regular maintenance. It was also observed in two hospitals that the receiving areas and recovery wards lacked equipped resuscitation trolleys for emergency preparedness. The reports highlighted that the funding of health services was a shared responsibility among the Ministry of Health, Ministry of Defense at Rwanda Military Hospital and the patients themselves through health insurance or cash payments.

The reports also revealed that the available staff included experienced general nurses, non-physician anesthetists, anesthesiologists, and surgeons who were committed to teach students, helping them to achieve their goals and objectives. However, none of the hospitals had trained and licensed perioperative nurses. The reports also highlighted that the hospitals had policies and procedures to guide the daily clinical activities. The theatre users committees were present in two out of three hospitals. These committees were composed of representatives of all cadres of surgical team and appointed hospital administration representatives. Their responsibilities included setting, implementing and monitoring operating theatres policies and procedures.

The clinical reports also revealed that all three referral hospitals were the centers for healthcare professionals' training for both formal and on-the-job trainings, which enhanced learning experiences for students among other personnel. There were opportunities for vertical training and specialization in disciplines of surgery, anaesthesia and perioperative nursing. These training facilities were also approved providers of continuous professional developments (CPD) by different professional councils. The availability of libraries, internet network and in-service trainings assisted the students and staff in accessing updated evidences.

The reports also highlighted the challenges of limited resources that still needed improvement for better surgical services and improved learning outcomes. Students observations revealed that the theatres layouts were not meeting the recommended standard requirements, $[9,14]$ The theatres had small corridors, small operating rooms that did not allow the appropriate housekeeping processes, the works of cleaning, and movements within the operating rooms. The students' observations revealed that the two of the three hospital's theatres had one sluice room for dirty utility and that the scrub-up areas were located in semirestricted area which should not be the case.

While ideally air exchange in operating room is recommended to be at least 15 times per hour, [15] the reports identified that the ventilation system in the two hospitals were not meeting the standards. The air was not filtered and exchanged as recommended and there were no scavenging systems for exhaled anesthetic gases threatening healthcare provider safety.

Against standards that there should be 1.5 to 2 PACU beds for each OR, $[16,17]$ the observations pointed out that all the three referral hospitals had few beds in PACU compared to number of operating rooms. It was also observed that there were frequent power outages without effective power backups, and shortage of flowing water supply from the taps was noted in two out of three hospitals. Use of stored water for surgical scrubbing is not recommended because it poises challenge to infection prevention and control practices.[18]

\section{Clinical leaning process}

There were rosters and daily allocations of staffs and students to ensure that at least each operating room had an experienced general nurse twined with a student. Students' allocation was guided by clinical objectives, operating theatre list, and hospital workload to immerse students in all activities. With regard to care delivery, the most important focus of perioperative nursing care is patient's safety and prevention of surgical site infections.[19] The immersion of students into care delivery and care coordination allowed the students to note the following observations:

The students reported the use of WHO surgical safety checklists. Other safety related items like patient securing straps, positioning devices, identification bracelet were also reported to be in use. WHO surgical safety checklists has been proven to be effective in reducing surgical related morbidity and mortality.[20]

The students also reported that they were involved in the scrubbing, circulating and assisting for the general, gynecology and obstetrics, orthopedic, Ear Nose Throat plastic and reconstructive, maxillofacial, endoscopic, urology, pediatric, thoracic, vascular and neurological surgeries. They also assisted in cardiac surgeries that were not routinely performed in Rwanda. They also participated in different techniques of anesthesia that were performed in these hospitals including local, regional and general anesthesia. The anesthesia care involved both pre anesthesia review and post anesthesia care. Moreover, students were also involved into instruments and equipment processing.

The students reported challenges with maintenance of equipment and operating room safety. Reports from two hospitals highlighted that unused equipment was kept in theatre and the resuscitation equipment were not regularly checked and replenished. The delicate, fragile instruments used in theatres were inappropriately 
handled and processed. In one hospital, Sodium Hypochlorite which is a cheap but corrosive solution[21] was reported as being used not in accordance with recommended guidelines of its use to clean stainless steel instruments, equipment and operating room leading to corrosion of surgical instruments and equipments.

In two out of three hospitals, wrapping of instruments did not follow the recommended number of layers and consistency of envelopes. This may expose the instruments to easy contamination and spread of infection to the patients. Furthermore, it was reported in one of the hospital that the sterilization indicator to differentiate processed from unprocessed instruments were not used. Additionally, waste segregation and waste disposal was only appropriate in one hospital although all the three hospitals had policy on waste management.

Regarding the coordination of care and operating theatre, every day, the unit managers allocated the nursing staff and appointed a senior nurse to coordinate each shift. The appointed person was responsible for receiving bookings, and ensuring smooth running of surgical procedures.

Nevertheless, the reports highlighted the overbooking of patients that might have contributed into patient cancellations due to time constraints. In one hospital, only one nurse was allocated in the operating room and was working as circulating nurse. In this case, the scrubbing role was performed by residents and medical students other than nurses, which is not in line with the standard practices.

It was also reported that the nurses working in operating theatre did not perform preoperative ward visits to assess patients prior to surgery according to standard practices, a fact that may jeopardize individualized patients' care. In this regard, further research is needed to find out the reasons why.

While the operating theatre is a special environment that needs additional precautions for better outcomes, it was also observed that the supporting staff from cleaning companies were not necessarily conversant with special operating theatre cleaning. Reports from two hospitals highlighted the inadequate orientation of new staff and students and this was reported to be limited to physical layout of theatre only.

\section{Outcomes}

Regarding learning experience, the, perioperative nurse students reported having benefited from the hospitals and achieved their clinical learning objectives despite some cited challenges.
The accreditation process of the teaching hospitals that requires continuous improvement in quality of care further enhanced learning process by providing conducive learning environment in addition to the existing infrastructures. The availability of many patients in hospitals aided the students to find different general and specialized surgical procedures as the learning opportunities. This helped the students to participate in management of diverse surgical conditions among patients of various ages. They managed to perform all perioperative nurses' role including preoperative assessment and patient preparation, intraoperative care (scrubbing and circulating) and postoperative management and follow up using nursing process.

The surgical teams from all three hospitals exhibited good attitude towards the students and showed willingness to teach. The team facilitated the students achieve the expected learning outcomes despite the challenges that none of the hospital had qualified trained perioperative nurses. The students benefited from expertise of one perioperative nurse specialist trainer who assisted them to overcome the challenges. This specialist had been hired through the collaboration between University of Rwanda, college of medicine and health sciences and Ministry of Health HRH program. She used to spend one day per week per hospital mentoring and teaching the perioperative students.

The students also used this opportunity to conduct inservice training to nurses working in these hospitals based on the identified training needs. The presentations included decontamination and sterilization process, safety, operating room environment cleaning and traffic control, and standard precautions among others. The master students also oriented and mentored the undergraduate and diploma students.

\section{Conclusion and recommendations}

Through the clinical practices, the students had great opportunities to sharpen their critical thinking and clinical reasoning skills. The surgical patients are at high risk for death and or other severe complications resulting from surgery and/or anesthesia. The complexity of perioperative care necessitates expertise and special trainings of the surgical team including perioperative nurses.

Therefore, Universities, Hospitals and Ministry of Health should continuously collaborate to promote the vertical education and specialization of their employees, particularly the surgical team and support the research in related fields of specialization to promote quality surgical healthcare through evidence-based practices. In this regard, the Universities should develop and deliver short courses of training and continuous 
Professional development programs on concepts related to operating theatre. Operating theatre designs should consider including the expertise of surgical team members including the perioperative nurse specialists who could help design new theatres or renovate the existing theatres and help meet international standards of modern theatres. Operating Room management team should enhance and monitor standards that promote infection prevention practice and safety in general. Training institutions should collaborate with the hospital to avail the qualified and experienced perioperative nurses for assisting students integrating theories into practice using the available resources.

\section{Acknowledgements}

We acknowledge the Ministry of Health, the University of Rwanda and HRH US faculty for their collaboration in establishing the specialty of perioperative nursing, and their immeasurable support to students during class and clinical teaching for achieving the objectives of this program.

We acknowledge University Teaching Hospital of Kigali, Rwanda Military Hospital and King Faisal Hospital for allowing our clinical allocation and availing resources (materials and human resources) that facilitated our learning. We thank the operating rooms staff for their contributions towards achieving clinical allocation objectives. We particularly thank the patients who trusted us to take care of them during the training period.

\section{References}

1. Ministry of Health Rwanda. Hman Resources for Health Strategic plan 2011-2016. 2011;5.

2. Uwizeye G, Mukamana D, Relf M, Rosa W, Kim MJ, Uwimana $P$, et al. Building Nursing and Midwifery Capacity Through Rwanda's Human Resources for Health Program. J Transcult Nurs. 2017;104365961770543.

3. Sharif F, Masoumi S. A qualitative study of nursing student experiences of clinical. 2005;7:1-7.

4. Motseki E 'Maliapa, Duma S. Student nurses' experiences of the operating theatre as a clinical learning environment in Lesotho. 2013;

5. Khoza LB. Nursing Students Perception of Clinical Learning Experiences. 2015;51:103-10.

6. Hamlin L, M.Richardson T, Davies M. Perioperative Nursing: Introductory Text. First. Mosby, Inc; 2009.

7. Meyer R, Prakoschandra R, Schalkwyk S Van. Student's perceptions of the operating rooms as clinical learning environment. 2014.
8. Goodman T, Cynthia S. Introduction to perioperative nursing. In: Essentials of perioperative nursing. 5th ed. Burlington, MA: Jones \& Bartlett Learning; 2014. p. 1-12.

9. Rothrock J, McEwen D. Alexander's Care Of the Patient in Surgery. 15th Editi. McEwen D, editor. Elsevier Inc; 2015. 1-12 p.

10. Alfaro-Lefevre R. critical Thinking, clinical reasoning, and clinical Judgement, a practical aproach. 6th editio. Elsevier Inc; 2017.

11. Mukamana D, Uwiyeze G, Sliney A. Nursing and Midwifery Education in Rwanda: Telling our Story. Rwanda J. 2015;2(2):9.

12. Harerimana A, Mtshali N., Mukamana D, Kimonyo J, Kayihura CN, Mugarura J. Historical Overview of Nursing and Midwifery Education and Nursing Workforce in Rwanda. Br J Med Heal Res. 2015;2(December).

13. Ikbal F. Beyond Accreditation : Issues in Healthcare Quality. Int J Res Found Hosp Healthc Adm. 2015;3(1):1-4.

14. Phillips N. Berry \& Kohn's Operating Room Technique. 15th ed. Elsevier Inc.; 2017. 167-200 p.

15. Spagnolo AM, Ottria G, Amicizia D, Perdelli F, Cristina ML. Operating theatre quality and prevention of surgical site infections. 2013;131-7.

16. Haret D, Ho E. Post Anesthesia Care Units. In: 2012 Operating Room Design Manual. American Society of Anesthesiologists; 2012.

17. Association of Anaesthetists of Great Britain and Ireland. AAGBI Safety Guideline: Immediate Post-anaesthesia Recovery 2013. Association of Anaesthetists of Great Britain and Ireland; 2013.

18. Miles LF, Scheinkestel CD, Downey GO. Environmental emergencies in theatre and critical care areas: Power failure, fire, and explosion. Contin Educ Anaesthesia, Crit Care Pain. 2015;15(2):78-83.

19. Sørensen E, Olsen I, Tewes M, Uhrenfeldt L. Perioperative nursing in public university hospitals: an ethnography. BMC Nurs. 2014;13:45.

20. Haynes AB, Weiser TG, Berry WR, Lipsitz SR, Breizat A-HS, Dellinger EP, et al. A Surgical Safety Checklist to Reduce Morbidity and Mortality in a Global Population. N Engl J Med. 2009;360(5):491-9.

21. Rutala WA, Weber DJ. Draft guideline for disinfection and sterilization in healthcare facilities. US-Centre Dis Control Prot. 2008;1-158. 